\title{
Angsthase soll kleinen Patienten Mut machen
}

- Bereits zum achten Mal begeben sich der Rabe Hexalus und die Augsburger Puppenkiste auf Kliniktour, um mit ihrem Mutmachstück „Das kleine Känguruh und der Angsthase" ein wenig Abwechslung und Freude in den Alltag kleiner Patienten zu bringen. Als Schirmherrin gab Bundesministerin Ilse Aigner am 12. März im Krankenhaus Agatharied im oberbayerischen Hausham den Startschuss.

Im Foyer des Krankenhauses konnten die kleinen Patienten eine halbe Stunde lang das kleine Känguruh und seine Freunde, die Springmaus, den Angsthasen und die Schlabberschlange, auf eine abenteuerliche Beerensuche begleiten und dabei ihre Krankheit eine Weile vergessen. Das Stück, in dem es um Freundschaft, Hilfsbereitschaft, Angst und Tapferkeit geht, basiert auf einer Geschichte des Kinderbuchautors Paul Maar. Von dem Kinderliedermacher Rolf Zuckowski stammt der Titel "Ich schaff' das schon“. Auf ein Honorar haben beide verzichtet.

Seit sieben Jahren organisiert und finanziert die Hexal Foundation die KlinikTour. Bundesweit sind dieses Jahr noch 23 Auftritte geplant. Kindern im Krankenhaus Mut zu machen sei das Ziel der Stiftung, betonte Helmut Fabry, Sprecher des Vorstandes der Hexal AG und Länderchef von Sandoz Deutschland. Mut beweist im Stück auch der Angsthase, dem die Freunde den glücklichen Ausgang ihres Abenteuers zu verdanken haben. Begeistert sind nicht nur die kleinen Zuschauer, sondern auch die Bundesministerin: „Ich wünsche mir, dass es diese Tournee noch lange gibt und dass viele Kinder den Zauber der Augsburger Puppenkiste erleben können."

\footnotetext{
- Birgit Matejka

Quelle: Pressekonferenz, Krankenhaus

Agatharied, 12. März 2010 (Veranstalter: Hexal Foundation)
}

\section{Kurz notiert}

Clopidogrel + ASS in einer Tablette - Das Unternehmen Sanofi-Aventis bietet den Thrombozytenaggregationshemmer Clopidogrelhydrogensulfat erstmals auch in Fixkombination mit ASS (75 mg/10o mg) an. DuoPlavin $^{\circledR}$ ist indiziert bei Patienten, die zur Prävention atherothrombotischer Ereignisse bereits die Einzelkomponenten erhalten.

Sanofi-Aventis

Kopflausmittel ohne kosmetische Zusatzstoffe - Das Kopflausmittel $\mathrm{Nyda}^{\circledR}$ wird jetzt auch als Nyda ${ }^{\circledR}$ sensitiv ohne Duft- und Zusatzstoffe angeboten. Die pedikulozide Wirkung beruht darauf, dass Dimeticon in die Atemwege der Läuse eindringt und sie verschließt. In einem In-vitro-Test zeigten beide Dimeticonpräparate eine stärkere lausabtötende Wirkung als andere handelsübliche Präparate (Heukelbach, BMC Dermatology 2009).

Pohl Boskamp

\section{Schmerzhafte Eingriffe bei Kindern}

\section{Inhalative Analgesie wirkt schnell und sanft}

— „In der Schmerztherapie bei Kindern bestehen immer noch Defizite“, sagte Prof. Michael Barthel, Bielefeld. Obwohl schmerzhafte Eingriffe in Kinder- und Notfallambulanzen zur Routine gehören, werden sie oft noch mit Festhaltemethoden durchgeführt. Doch gerade bei kleinen Kindern ist eine effektive Analgesie zwingend erforderlich, um Verhaltensauffälligkeiten und gesteigertes Schmerzerleben bei späteren Eingriffen zu vermeiden.

Eine innovative inhalative Analgesie, die schnell und sanft wirkt, ist eine Fertigmischung aus 50\% Distickstoffmonoxid und Sauerstoff (Livopan ${ }^{\circledR}$ ). „Gerade für Nichtanästhesisten steht damit eine Behandlungsoption zur Verfügung, die keine erhöhte Komplikations- oder Nebenwirkungsrate aufweist“, so Barthel. Die mittelstarke analgetische und zugleich beruhigende Wirkung setzt nach zwei bis drei Minuten ein. Sie bietet sich z. B. an bei Materialentfernung, Wundversorgung, geschlossener Reposition, Reposition einer Paraphimose, Fremdkörperentfernung, Gelenkpunktion und Entfernen einer Warze. „Mit Livopan ${ }^{\circledR}$ lassen sich bei kleinen Eingriffen Vollnarkosen meist vermeiden", so Barthel. Seine Erfahrung bei mehr als 100 Patienten von 10 Monaten bis 15 Jahren habe gezeigt, dass das Verfahren absolut sicher ist. „Bei Patienten, Eltern und Personal fanden sich eine hohe Akzeptanz und gute Resonanz", so Barthel.

Das Arzneimittel wird über eine Maske mit einem Demandventil appliziert. Der Patient bleibt ansprechbar und die Schutzreflexe sind nicht beeinträchtigt.

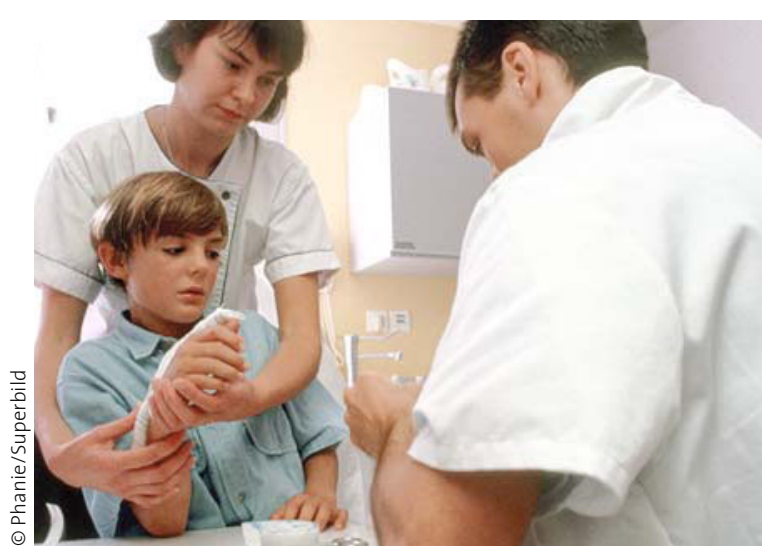

Nicht mehr zeitgemäß: die Festhaltemethode bei schmerzhaften Eingriffen.

\footnotetext{
- Dr. med. Peter Stiefelhagen

Quelle: Workshop, Tagung der Süddt. Gesellschaft für Kinder- und Jugendmedizin,
} Marburg, 19. März 2010 (Veranstalter: Linde) 